\title{
Estilo interactivo durante la lectura de cuentos infantiles de contenido engañoso
}

\author{
Ma José Rabazo Méndez y \\ Juan Manuel Moreno Manso
}

Departamento de Psicología y Sociología de la Educación, Universidad de Extremadura, Badajoz

España

jmmanso@unex.es 


\section{Resumen}

El propósito de este artículo es, por una parte, analizar la frecuencia con que aparecen las referencias a los estados mentales en cuatro cuentos infantiles con contenido engaño y, por otra, describir el carácter de las interrupciones maternas en una actividad cotidiana de lectura de cuentos. Según el contenido, las interrupciones se han agrupado en torno a cuatro tipos de actos de habla: de contenido asertivo, de contenido interrogativo, contenido directivo y contenido expresivo. Las medidas lingüísticas maternas recogidas han sido la frecuencia y la diversidad en el uso de lenguaje de referencia mental según distintas categoría: términos perceptivos, emocionales, volitivos y cognitivos, según el modelo de categorización de Bretherton y Beeghly (1982) utilizadas en cada tipo de actos de habla. Se presentan los datos pertenecientes a una muestra exploratoria formada por 18 díadas de madres-hijos.

Las conclusiones más destacadas son las siguientes: Los cuentos analizados contienen abundantes referencias a las relaciones entre la realidad, las creencias y la conducta de los personajes; las madres interrumpen la lectura ante un contenido de engaño aparecido en el relato; en una misma interrupción pueden observarse varios tipos de actos de habla; las interrupciones de tipo asertivo y de tipo expresivo son las más utilizadas por las madres durante la lectura y emplean un vocabulario de tipo mentalista y de tipo emocional en el mayor número de casos para resaltar o aclarar algunas de las situaciones relacionadas con la trama de engaño.

Palabras Clave: lectura, cuentos, actos de habla, vocabulario mentalista, teoría de la mente. 


\section{Introducción}

La habilidad para comprender y atribuir en los otros estados mentales como las creencias, emociones, deseos, intenciones y conocimientos, que pueden ser distintos a los nuestros y distintos también del estado real de los hechos, es una capacidad muy importante del desarrollo socio-emocional de los niños. A través de esta capacidad los niños pueden comprender las acciones de las personas como producto de estados mentales internos (creencias, deseos, etc.) y por tanto pueden realizar predicciones sobre el comportamiento humano. Esta comprensión resulta fundamental para entender la conducta de los demás y coordinarla con la nuestra, haciendo así posible la interacción social (Villanueva, Clemente y García, 2002) y posibilita la realización de conductas tendentes a generar en nosotros un estado mental que no corresponde con la realidad (creencia falsa) y además intenta sacar partido de ello (Sotillo y Rivière, 2001).

Distinción entre conducta accidental y conducta intencional, entre deseos y realidad y entre verdad y engaño, regulación emocional, son aspectos que son posibles gracias a esta comprensión. ¿Pero cómo llegan los niños a dar significado a lo que hacen las personas? ¿Cuál es la contribución de los cuidadores principales a la comprensión de la mente y a través de qué mecanismos ofrecen su "andamiaje?

El papel fundamental de la interacción social en el desarrollo cognitivo temprano ha sido demostrado en numerosos estudios (Vygotsky, 1978; Wood, Bruner y Ross, 1976; Werstch, 1979; Rogoff, 1990). Esta línea de investigación destaca las formas de interacción entre niños y mayores en contextos sociales de aprendizaje como facilitadoras del desarrollo de las funciones psicológicas superiores de los niños, entre ellas el acceso al mundo mental o la comprensión de la mente.

Diversos autores han contribuido a una interpretación cultural del desarrollo de los estados mentales. Para Cole (1995), la mente se ha desarrollado entre y por medio de la cultura, cuyos productos actuarían de recipiente material del pensamiento sobre los demás.

Montgomery (1997) plantea que nuestra concepción y nuestro acceso a nuestros propios estados mentales y a los de los demás es un proceso embebido en la adquisición de las reglas que gobiernan el uso de las expresiones lingüísticas sobre la mente. No es posible 
aprender el significado de los términos mentales simplemente mediante la introspección, a partir de nuestra experiencia interna. El criterio para un uso correcto de los términos mentales es necesariamente público, lo que implica un proceso social de construcción de su significado. Autores como Lillard (1998) plantean que los padres establecen un discurso estructurado acerca de los aspectos representacionales de la mente en un proceso esencialmente educativo y coherente con las características culturales de su comunidad de referencia.

Garfield y cols. (2001) ofrecen un marco teórico por el cual el desarrollo de los estados mentales sería dependiente conjuntamente del lenguaje y de la experiencia social. Su desarrollo sería el resultado de la conjunción de la adquisición lingüística y de la progresión en la comprensión social del niño adquiridas a través de las conversaciones y las interacciones con los demás.

Bruner (1990, 1997), Carrithers (1991), Nelson (1981) conceden una importancia capital a la "narratividad" como proceso a través del cual el niño puede comprender a los personajes de una acción, sus expectativas, intenciones, creencias, motivos y las consecuencias de sus acciones a partir de sus estados mentales. Esos guiones o estructuras narrativas ayudan a organizar el conocimiento y permiten crear expectativas sobre la secuencia de los acontecimientos y permitirían al niño pensar en cualquier actividad como una secuencia coordinada de sucesos que incluirían dos componentes, lo que Bruner (1986) denomina el componente conductual y los argumentos de la acción: el agente, la situación, los instrumentos, etc. y el interno o intencional de los acontecimientos: lo que se conoce o no, lo que se piensa o no, lo que se siente o no.

Autores como Dunn, Bretherton y Munn (1987), Furrow, Moore, Davidge y Chiasson (1992), Moore, Furrow, Chiasson y Patriquin (1994), Stern (1985) han investigado en el área de la producción, adquisición y comprensión del lenguaje mentalista la influencia del lenguaje adulto. Sin embargo, a la luz de los resultados no se pueden establecer relaciones causales entre el discurso materno y las capacidades mentales en los niños.

Recientemente se ha empezado a examinarse la relación entre el habla adulta y el desarrollo de competencias mentalistas en torno a la actividad conjunta de la lectura de cuentos, situación interactiva más tradicional en todos los hogares, recurso que, por otra parte, ha sido investigado en relación a los procesos de categorización y adquisición lingüística, desarrollo alfabético (Bruner, 1975, 1986; Ninio, 1983; Baudier, Fontaine y Pecheux, 1997; Del Río y García, 1996; Bornstein y Haynes, 1998; Peralta y Salsa, 2001). La lectura de material ilus- 
trado es una de las situaciones diádicas más comunes en las que se hace explícito el rol tutorial del adulto (Hoff.-Ginsberg, 1991)

Parece ser que, durante la lectura de cuentos, los adultos suelen producir enunciados que se refieren a personas, objetos y lugares que no están físicamente presentes en el contexto inmediato (Deleau, Gandon y Taburet, 1993) y además sus producciones suelen tener mayor nivel de abstracción comparadas con las producciones durante juegos de modelado (Sorsby y Martlew, 1991).

Bruner $(1975,1983)$ argumentaba que la lectura conjunta de cuentos implica cierto nivel de intersubjetividad entre los participantes, dado que los objetos a los que se atiende son compartidos, al igual que las palabras para referirse a ellos y el conocimiento implicado en esa comunicación. De esta forma, esta actividad se convierte, al igual que los primeros juegos infantiles, en una fuente de oportunidades para el "andamiaje" en las interacciones entre los niños y miembros más expertos de su cultura que capacitan al niño para actuar en un nivel más avanzado que en su actividad independiente y es precisamente en el contexto conversacional entre el adulto que lee y el niño que interrumpe la lectura donde se produce el andamiaje; el niño puede estar confundido sobre la acción y aventurar interpretaciones, ante lo cual el adulto se esfuerza por facilitar su comprensión ofreciendo explicaciones. De esta manera ambos, conjuntamente, estructuran la interacción, unas díadas mejor que otras, de manera que el niño va aumentando su grado de control y responsabilidad sobre el proceso a medida que su competencia va aumentando (Rogoff, 1989).

Se plantea como objetivo del presente trabajo analizar la frecuencia de aparición de vocabulario mentalista en cuatro cuentos de hadas de contenido engañoso así como describir el tipo de contenido de los actos de habla utilizados por las madres durante la lectura y comentario de varios cuentos de ese tipo de contenido a sus hijos, así como analizar la diversidad en el uso de lenguaje de referencia mental atendiendo a términos perceptivos, emocionales, volitivos y cognitivos, según el modelo de categorización de Bretherton y Beeghly (1982). 


\section{Método}

Sujetos

Se trabajó con una muestra de 18 díadas de madres y sus hijos residentes en Badajoz. Las madres participaron de forma voluntaria y se estableció el contacto a través del alumnado de magisterio de la Facultad de Educación. Los niños tenían 3 años $(\mathrm{N}=5), 4(\mathrm{~N}=7)$ y $5(\mathrm{~N}=6)$, situándose la media de edad en torno a 54 meses (4,5 años). De ellos, 10 eran niñas y 8 eran niños. La edad de las madres se situó entre los 23 y los 39 años, siendo la media 32,27. El nivel socioeconómico de las familias era de medio-alto.

\section{Instrumentos}

Los instrumentos que hemos utilizado han sido cuatro cuentos (Hansel y Gretel, El Gato con Botas, Caperucita Roja y Blancanieves) de contenido engañoso.

En estos cuentos se abordan las formas que pueden adquirir el engaño, la trampa y la mentira, se describen los procesos de planificación de los engaños y las mentiras y las consecuencias que se derivan tanto para el embustero como para la víctima. Este tipo de contenidos aparecidos en los cuentos elicitan en las madres comentarios, expansiones explicativas sobre los contenidos así como conclusiones de tipo moral referidas a las fechorías de los personajes y a sus consecuencias o dirigiendo la atención del niño hacia aquellos aspectos de las historia que aportan información clave para desenmascarar al mentiroso

\section{Procedimiento}

El criterio para elegir estos cuentos ha sido que cumplen los requisitos que deben concurrir cuando hablamos de engaño: 1) dos sujetos que compiten; uno de ellos - el que miente - tiene intención de engañar al otro; 2) quien miente usa estrategias manipulativas del estado mental del otro, dando al competidor información falsa (u ocultándole la verdadera) para crear en él una creencia falsa sobre un contenido; 3) como consecuencia, quien miente consigue su objetivo: proporciona al otro una creencia falsa; y 4) como consecuencia, quien miente consigue su objetivo: proporciona al otro una creencia falsa (Bennett, 1976) 
Una vez seleccionados los cuentos, se confeccionaron unos protocolos para la recogida de la información sobre el vocabulario mentalista según la clasificación de Bretherton y Beeghly (1982).

Dos personas diferentes, pertenecientes al equipo de investigación leían los cuentos y anotaban las referencias al vocabulario mentalista en la hoja de registro.

Por otra parte, la consigna dada a las madres era "leer y comentar (si es que lo hace) un cuento a su hijo o a su hija de la forma en que habitualmente lo hace. Usted puede leer el cuento las veces que estime oportuno antes de leer o contar ese cuento a su hijo". La lectura del cuento se realizó en el hogar de los niños y la sesión fue grabada en video por un alumno (conocido de la familia).

El contenido de las grabaciones de la lectura de cuentos fue trascrito a protocolos y codificados para su posterior análisis.

\section{Análisis de los datos}

\section{$\underline{\text { Sistema de codificación }}$}

El contenido del vocabulario de los cuentos se clasificó con arreglo al modelo de categorización empleado por Bretherton y Beeghly (1982) quienes distinguen entre términos referidos a procesos cognitivos (pensar, imaginar, saber...), afectivos (enfadarse, alegrarse, triste, contento...), volitivos (querer, desear...) y perceptivos (mirar, ver, fijarse...). Todos los términos de tipo cognitivo aparecidos en las transcripciones fueron computados independientemente de su uso.

En cuanto a las interrupciones de las madres durante la lectura, se clasificaron en cuatro tipos de Actos de Habla:

1. Asertivo. Sirve para dar información al destinatario.

2. Interrogativo. Su propósito es obtener información del destinatario.

3. Directivo. Su fin es lograr que el destinatario lleve a cabo una acción.

4. Expresivo. Manifiesta la subjetividad y el punto de vista del emisor. 


\section{Fiabilidad del sistema de codificación}

Dos lectores independientes efectuaron el recuento de los términos mentalistas aparecidos en los cuentos. En la tabla 2 aparece los valores del estadístico Kappa de Cohen que indican buen grado de acuerdo en general.

Respecto a la evaluación de las interrupciones maternas, el grado de acuerdo entre observadores sobre las categorías se realizó a partir de la codificación y recuento independiente por parte de una segunda persona del 30\% de las transcripciones. En la tabla 1 se presentan las categorías codificadas, ejemplos de cada una de ellas y los valores de fiabilidad interjueces a partir del estadístico Kappa de Cohen, que indican un buen grado de acuerdo general.

Tabla 1. Acuerdo interjueces sobre los tipos de Actos de Habla

\begin{tabular}{|c|c|c|}
\hline Categoría & Ejemplos & Kappa \\
\hline Asertivo & $\begin{array}{l}\text { - ¿Qué mentiroso! La quiere engañar } \\
\text { - } \quad \text { El gato está poniendo una trampa } \\
\text { - } \quad \text { iQué mala! Quiere comerse a los niños }\end{array}$ & .94 \\
\hline Interrogativo & $\begin{array}{l}\text { - ¿Y quién será el marqués de Carabás? } \\
\text { - ¿Para quién será? }\end{array}$ & .95 \\
\hline Directivo & $\begin{array}{l}\text { - Vamos a ver que le dice } \\
\text { - ¡Oh mira! Una casita de golosinas }\end{array}$ & .91 \\
\hline Expresivo & $\begin{array}{l}\text { - ¿Qué mala la madrastra! } \\
\text { - Qué listo el gato! } \\
\text { - ¿Qué listo Hansel! }\end{array}$ & .96 \\
\hline
\end{tabular}

\section{Resultados}

Uno de los propósitos de la investigación fue analizar el contenido de vocabulario mentalista aparecidos en cuatro cuentos infantiles. Se efectuó un análisis de frecuencias. En la tabla 2 aparecen los resultados de las frecuencias así como el grado de acuerdo interjueces. Como podemos observar son los términos relacionados con los procesos perceptivos los que destacan sobre el resto de cognitivos, seguidos, de términos de contenido afectivo y cognitivo. Una mención frecuente a las emociones puede ayudar a los niños a comprender las características de los sucesos mentales. La referencia a los deseos, términos de contenido volitivos, va detrás de la aparición de términos cognitivo en contra de lo que sucede en el discurso natural, según Bartsch y Wellman (1995). 
Tabla 2. Frecuencia de vocabulario de tipo cognitivo* y acuerdo interjueces

\begin{tabular}{|c|c|c|c|c|c|c|c|c|c|}
\hline & $\begin{array}{c}\text { Caperucita } \\
\text { Roja }\end{array}$ & Kappa & Blancanieves & Kappa & $\begin{array}{c}\text { Hansel } \\
\text { y Gre- } \\
\text { tel }\end{array}$ & Kappa & $\begin{array}{c}\text { El } \\
\text { Gato } \\
\text { con } \\
\text { Botas }\end{array}$ & Kappa & Total \\
\hline Cognitivos & 7 & .91 & 6 & .90 & 15 & .88 & 9 & .89 & 37 \\
\hline Afectivos & 8 & .89 & 11 & .91 & 12 & .90 & 11 & .91 & 42 \\
\hline Percepctivos & 11 & .90 & 15 & .92 & 11 & .93 & 18 & .93 & 55 \\
\hline Volitivos & 7 & .93 & 8 & .90 & 6 & .91 & 5 & .90 & 26 \\
\hline $\begin{array}{c}\text { Total cogni- } \\
\text { tivos }\end{array}$ & \multicolumn{3}{|c|}{40} \\
\hline
\end{tabular}

Otro de nuestros objetivos fue describir el carácter de las interrupciones maternas durante la lectura de cuentos. Las medidas lingüísticas maternas recogidas fueron la frecuencia y la diversidad en el uso de lenguaje de referencia mental según distintas categoría: términos perceptivos, emocionales, volitivos y cognitivos, según el modelo de categorización de Bretherton y Beeghly (1982)

En la tabla 3 se presenta el promedio de las interrupciones llevadas a cabo por las madres durante la lectura del cuento. Como podemos observar la media de las interrupciones aumenta a medida que aumenta la extensión de los cuentos.

Tabla 3. Promedio de las interrupciones

\begin{tabular}{|l|l|l|l|l|}
\hline Título del cuento & Caperucita Roja & Blancanieves & Hansel y Cretel & El Gato con Botas \\
\hline Media & 6,8 & 8,6 & 11,3 & 10,5 \\
\hline $\mathrm{N}=18$ & \multicolumn{2}{|l}{} \\
\hline
\end{tabular}

En la tabla 4 quedan reflejadas las medias y el porcentaje de los diferentes tipos de actos de habla y en la tabla 5 se resaltan el tipo de vocabulario cognitivo en función del contenido del acto de habla. Comentamos a continuación cada uno de los contenidos de los actos de habla.

Contenido asertivo. El acto de habla de contenido asertivo sirve para dar información. A través del mismo la madre expande el contenido explícito de la narración con el obje- 
tivo de facilitar la comprensión, interpretando e infiriendo el significado de los acontecimientos y de los puntos de vista de los personajes.

Estos actos de habla hacen alusión a:

- Los estados de los personajes: Como es pequeña tiene miedo...su hermano la consuela (en Hansel y Gretel).

- A los deseos: El lobo tiene hambre, quiere comérsela (en Caperucita Roja).

- A las intenciones: Se viste de abuelita para engañar a Caperucita (en Caperucita Roja).

- A las creencias: Quiere que su abuela crea que es Caperucita (en Caperucita Roja) o Le lleva otro corazón para que crea que es el de Blancanieves (en Blancanieves).

- Destaca acciones: La quiere engañar (en Caperucita Roja) o Va a llevarle otro corazón (en Blancanieves) o Dice eso para que lo oiga el rey (en el Gato con Botas).

Como podemos observar en la tabla 5 , del 55,84\% de las expansiones asertivas, el $40,6 \%$ incluyen un tipo de vocabulario mentalista y es donde se tratan los contenidos de engaño del cuento. Hacen referencia a los pensamientos y creencias de los personajes así como a sus intenciones de engañar.

- ¿Qué mentiroso! La quiere engañar (en Caperucita)

- Este ogro no sabe que el gato es muy listo (en el Gato con Botas)

- Está pensando en otro plan (en Hansel y Gretel)

- Quiere que la abuela crea que es Caperucita (en Caperucita Roja)

Los Actos de Habla de contenido asertivo aparecen como aclaración o descripción de alguna eventualidad de la acción de los personajes a continuación de Actos de Habla de tipo:

- Exclamativo ;Qué mentiroso! La quiere engañar ( en Caperucita Roja)

- Directivo Mira lo que hace: se viste de abuelita (en Caperucita Roja)

- Interrogativo ¿Y por qué dirá eso? Quiere que su abuelita crea ...(en Caperucita Roja) 
Contenido Interrogativo. Este tipo de contenido tiene el propósito de obtener información del destinatario o hacerle reflexionar sobre algún aspecto relacionado con la trama de engaño.

El 37,43\% de las interrupciones son de este tipo, aunque, según hemos podido comprobar, las madres, en la situación de lectura, no suelen dejar tiempo para que el niño responda, sino que a continuación contestan ellas utilizando una expansión asertiva:

- ¿Y por qué dirá eso? (pequeño silencio) Ah, quiere que su abuela crea que es Caperucita. (Pregunta que hace referencia a los personajes)

- El rey quiere saber quién es el marqués de Carabás. Y tú, ¿quieres saberlo? (Pregunta referida al niño)

Del 37,43\% de las interrogaciones efectuadas, en el 34,23\% de todas ellas se utiliza una variedad léxica de tipo cognitivo y, al igual que los actos de habla de contenido asertivo, está relacionada con los pensamientos y creencias de los personajes, dando, a través de la pregunta, la oportunidad al niño de abordar la causalidad de la acción, los distintos sentimientos de los personajes o para explorar los efectos de las creencias en la conducta.

Contenido directivo. La utilización de los actos de habla de tipo directivo tiene el objetivo de llamar la atención del niño sobre algún aspecto de la acción de los personajes.

En el total de las interrupciones de este tipo, en el 40,93\%, se utiliza un vocabulario de tipo perceptivo y focalizando la atención del niño sobre el contenido engañoso de la historia:

- Fíjate, le enseña un hueso (en Hansel y Gretel)

Contenido expresivo. Los actos de habla de contenido expresivo tienen la función de compartir los estados mentales y enfatizan algún punto importante. Expresan una valoración del punto de vista del adulto:

- Qué listo este gato! (en el gato con botas)

- ¿Qué mala la bruja! (en Blancanieves)

- iQue valiente es Hansel! (en Hansel y Gretel) 
En el total de las interrupciones de tipo expresivo, el $88,36 \%$, se utiliza un vocabulario calificativo de tipo afectivo- emotivo. A través de este etiquetado el adulto socializa y da nombre a las emociones que cree correctas.

Sobre este tipo de interrupciones hay que resaltar dos aspectos fundamentales: en primer lugar, todos los personajes llevan a cabo acciones de engaño, trampa o mentira; en segundo lugar, la valoración que hace el adulto del personaje engañoso adopta dos posturas contrarias: De sabiduría, de listeza, de astucia o de maldad; así el gato con botas miente y es listo mientras que el lobo miente pero es malvado. Tanto en un caso como otro se trata de capacidades que presuponen sofisticados sistemas de simulación con fines adaptativos.

Tabla 4. Medias y Porcentajes del tipo de Actos de Habla

\begin{tabular}{|c|c|c|c|c|c|}
\hline & $\begin{array}{l}\text { Caperucita } \\
\text { Roja }\end{array}$ & Blancanieves & $\begin{array}{c}\text { Hansel y } \\
\text { Cretel }\end{array}$ & $\begin{array}{l}\text { El Gato con } \\
\text { Botas }\end{array}$ & Total \\
\hline Asertivo & $5,2(76,47 \%)$ & $7,3(84,88 \%)$ & $6,7(59,29 \%)$ & $9,2(87,61 \%)$ & $55,84 \%$ \\
\hline Interrogativo & $3,2(47,05 \%)$ & $2,4(27,90 \%)$ & $3,5(30,97 \%)$ & $4,6(43,80 \%)$ & $37,43 \%$ \\
\hline Directivo & $3,8(55,88 \%)$ & $3,4(39,53 \%)$ & $3,2(28,31 \%)$ & $4,2(40 \%)$ & $40,93 \%$ \\
\hline Expresivo & $6,7(98,52 \%)$ & $7,8(90,69 \%)$ & $9,2(81,41 \%)$ & $8,7(82,85 \%)$ & $88,36 \%$ \\
\hline \multicolumn{6}{|c|}{$\begin{array}{l}\mathrm{N}=18 \\
\text { Nota: El total de los porcentajes de las interrupciones no suma } 100 \text { porque en una mis- } \\
\text { ma interrupción se pueden observar varios tipos de actos de habla. }\end{array}$} \\
\hline
\end{tabular}

Tabla 5. Vocabulario de tipo cognitivo

\begin{tabular}{|l|c|c|c|c|}
\hline & $\begin{array}{c}\text { Asertivo } \\
(55,84 \%)\end{array}$ & $\begin{array}{c}\text { Interrogativo } \\
(37,43 \%)\end{array}$ & $\begin{array}{c}\text { Directivo } \\
(40,93 \%)\end{array}$ & $\begin{array}{c}\text { Expresivo } \\
(88,36 \%)\end{array}$ \\
\hline $\begin{array}{l}\text { Procesos cognitivos } \\
\text { (pensar imaginar, } \\
\text { engañar, creer, sa- } \\
\text { ber) }\end{array}$ & $26,2 \%$ & $12,93 \%$ & - & - \\
\hline $\begin{array}{l}\text { Afectivos (enfadar- } \\
\text { se, alegrarse, triste, } \\
\text { contento...) }\end{array}$ & $5,3 \%$ & - & - & $88,36 \%$ \\
\hline $\begin{array}{l}\text { Volitivos (querer, } \\
\text { desear...) }\end{array}$ & 14,4 & $21,3 \%$ & - & - \\
\hline $\begin{array}{l}\text { Perceptivos (mirar, } \\
\text { ver, fijarse....) }\end{array}$ & $9,94 \%$ & $3,2 \%$ & $40,93 \%$ & - \\
\hline
\end{tabular}




\section{Discusión y Conclusiones}

En primer lugar, quisiéramos poner de manifiesto que entendemos el proceso de lectura de cuentos como un proceso interactivo, donde la contribución del cuidador se adapta y es sensible al interés y al comportamiento mostrado por el niño y es en esa línea donde se enmarca nuestra investigación, a pesar de que en este artículo sólo analicemos el tipo de interrupciones adultas durante la actividad.

De los resultados obtenidos se puede constatar que las referencias a los estados mentales son frecuentes en los cuentos infantiles con contenido de engaño y hacen mención explícita a los pensamientos, sentimientos e intenciones de los personajes. Los términos con mayor frecuencia de aparición han sido los de tipo afectivo, seguidos de los cognitivos y de las referencias a los deseos. Estos resultados apoyan los encontrados por Dyer y cols. (2000). La aparición de este tipo de vocabulario mentalista puede ser un potencial muy importante a la hora de proporcionar la comprensión de las relaciones entre la realidad, las creencias y la conducta.

Las madres interrumpen su lectura y comentan algún aspecto del cuento al encontrarse ante las situaciones de contenido engañoso. Hay que resaltar que las conductas de engaño y mentira aparecen en situaciones de interacción social intencionada, utilizan habilidades relacionadas con la realización de inferencias mentalistas (de teoría de la mente), implican diferenciar la representación y el mundo, también implican diferenciar la representación propia y la ajena. Los análisis secuenciales de los diálogos evidencian conductas de coordinación social como la complementación y la expansión.

Del análisis de frecuencias se desprende que de todas las interrupciones de la madre durante la lectura, destacan por su mayor porcentaje las de contenido expresivo seguidas de las de contenido asertivo.

Existen diferencias en cuanto al objetivo de las interrupciones. A través de las expansiones asertivas las madres tratan de facilitar la comprensión de la trama engañosa y a través de las interrupciones de tipo expresivo tratan de etiquetar al personaje en función de las intenciones y a través de este etiquetado socializar al niño en los valores culturales y en los patrones de conducta. 
Se evidencia una mayor frecuencia de aparición de vocabulario cognitivo y afectivo, posiblemente con la finalidad de ayudar al niño a comprender las características de los sucesos mentales a través de una contínua asociación con los estados cognitivos.

En este sentido, Vygotsky (1986) enfatizaba que durante la lectura de cuentos los cuidadores primero atraen la atención del niño hacia el vocabulario más familiar presente en el relato para después introducir nuevos términos desconocidos o no dominados por el niño.

Nos parece interesante señalar la importancia de este tipo de estudios de cara a la intervención psicoeducativa con personas que presenten necesidades educativas especiales y tengan dificultades para manejarse en la compleja red de relaciones interactivas: Conocer el tipo de estrategias utilizadas por los adultos de forma implícita, en este caso, a través de una "lectura dialogada" cuando detectan un contenido engañoso para contribuir a su comprensión, puede ayudarnos a los profesionales para adaptar los cuentos en los puntos en que se ha encontrado que las madres interrumpen la lectura.

El empleo de ilustraciones y la utilización de los "bocadillos" con dibujitos de lo que la gente tiene en la cabeza así como la instrucción a sus cuidadores en el uso de las estrategias citadas podría contribuir a la mejora en la predicción de la acción de un personaje y a la atribución de la creencia de un personaje en relación a un estado de hechos que ha cambiado en su ausencia.

En una segunda parte del estudio se analizan las diferencias individuales encontradas en la compresión infantil de la creencia falsa en función del contenido de las interrupciones maternas con la finalidad de argumentar la posible importancia del input lingüístico adulto, estructurado en formas particulares, para la elaboración de la teoría de la mente de los niños.

\section{Referencias}

Adrián, J. y Clemente, R. (2002). Relaciones entre el lenguaje materno sobre términos verbales cognitivos y la comprensión infantil de estados mentales. Tesis doctoral inédita. Universitat Jaume I

Astington, J. W. y Gopnik, A. (1991). Theoretical explanations of children's understanding on the mind. British Journal of Developmental Psychology, 9, 7-31 
Baron-Cohen, S. (1995). The eye-direction detector (EDD) and the shared attentio Mechanism (SAM): Twocases for evolutionay psychology. En C. Moore y P. Dunham (Eds.), Joint attention: Its origins and role in development. Hillsdale, HJ: Erlbaum

Baron-Cohen, S. (1987). The autistic child's theory of mind: A case of specific developmental delay. Journal of Child Psychology and Psychiatry, 30, 285-297

Baron-Cohen, S., Jolliffe, T., Mortimore, T, y Robertson, M. (1997). Another advanced test of theory of mind: Evidence from very high functioning adults with Autism or Asperger syndrome. Journal of Child Psychology and Psychiatry, 38, 813-822

Baron-Cohen, S., Leslei, A. M., Frith, U. (1985). Does the autistic child have a "theory of mind? Cognition, 21, 37-46

Baron-Cohen, S., Leslei, A. M., Frith, U. (1986). Mechanical, behavioral and intentional understanding of picture stories in autistic children. British Journal of Developmental Psychology, 4, 113-125

Bartsch, K. y Wellman, H. M. (1995). Children talk about the mind. New York: Oxford University Press

Baudier, A., Fontaine, A. M. y Pêcheux, M. G. (1997). Etayage maternal de l'atterntion dans une situation de lectura chez des enfants de 1 à 3 ans. Enfance, 2, 229-245

Bennett, J. (1976). Linguistic behaviour. Cambridge: Cambirdge University Press

Bettelheim, B. (1999). Psicoanálisis de los cuentos de hadas. Barcelona: Crítica

Bornstein, M. y Haynes, M. (1998). Vocabulary competente in early childhood: measurement, latant, construct, and predicative validity. Child Development, 69, 654-305.

Bretherton, I. y Beeghly, M. (1982). Talking about internal states: The acquisition of an explicit theory of mind. Deelopmental Psychology, 18, 906-921.

Bruner, J. (1975). The ontogenesis of speech acts. Journal of Child Language, 2, 1-19

Bruner, J. (1983). Child's talk: Learning to use language. New York: Norton

Bruner, J. (1986). El habla del niño. Barcelona: Paidós

Bruner, J. (1990). Acts of meaning. Cambridge, MA: Harvard University Press 
Bruner, J. (1990). Acts on meaning. Cambridge, MA: Harvard University Press

Bruner, J. (1995). Comentary. Human Development, 38, 203-213

Bruner, J. (1997). La educación, puerta de la cultura. Madrid: Visor

Carrithers, M. (1991). Narrativity: Mindreading and making societies. En A. Whiten (Ed.), Natural theories of mind: Evolution, development and simulation of everyday mindreading (pp. 305-318). Oxford: Brasil Blackwell

Carrithers, M. (1991). Narrativity: Mindreading and making socities. En A. Whiten (Ed.), Natural theories of mind. Evolution, development and simulation of evereday mindreaning, (pp. 305-318). Oxford: Basil Blackwell

Cole, M. (1995). La cultura in una teoria della comunicazione della mente. En O. Liverta Sempio \& A. Marchetti (Eds.), Il pensiero dell'altro. Contesto, conoscenza e teorie della mente (pp. 97-124). Milano: Rafaello Cortina Editore

Dalke, D. E. (1995). Explaining young children's difficulty on the false belief task: Representational deficits on context-sensitivity knowledge? British Journal of Develomental psychology, 13 (3), 209-222

Del Río, M. y García, M. (1996). Una aproximación al análisis de los intercambios comunicativos y lingüísticos entre niños pequeños y adultos. Infancia y Aprendizaje, 75, 3-20

Deleau, M., Gandon, E., y Taburet, V. (1993). Semiotic mediation in guiding interactions with young children: the role of context and communication handicap on distanciation in adult discourse. European Journal of Psychology of Education, 8, 473-486

Dennet, D. C. (1991). La actitud intencional. Barcelona: Gedisa

Dunn, J. (1988). The beginnings of social understanding. Cambridge, MA: Harvard University Press

Dunn, J. (1991). Undertanding others: Evidence from naturalistic studies of children. En En A. Whiten (Ed.), Natural theories of mind. Evolution, development and simulation of evereday mindreaning, (pp. 51-62). Oxford: Basil Blackwell

Dunn, J., Bretherton, I. y Munn, P. (1987). Conversations about feeling states between mother and their young children. Developmental Psychology, 23, 132-139 
Dyer, J. R. Shatz, M. Wellman, H. M. (2000). Young childre's storybooks as a source of mental state information. Cognitive Development, 15, 17-37

Flavell, J. H., y Miller, P. (1998). Social cognition. En W. Damon, D. Kuhn y R. S. Siegler (Eds.), Handbook of Chile Psychology, Vol. III. Cognition, Perception and Language, 851-898. New York: Wiley \& Sons

Furrow, D.,Moore, C., Davidge, J. y Chiasson, L. (1992). Mental terms in mothers`and children's speech: Similarities and relationship. Journal of child Language, 19, 617-631

Garfield y cols. (2001). Social Cognition, Language acquisition and The Development of the Theory of Mind. Mind and Language, 16, 494-541

Goicoechea, M. A. y Clemente, R. A. (2003). El Discurso materno y la contribución a la comprensión de las emociones (pp. 107-115). En Fajardo y cols. (Eds), Infancia y Adolescencia: Desarrollo psicológico y propuestas de intervención. Badajoz: Psicoex

Happé. F. G. E., Winner, E., y Browell, H. (1998). The getting of wisdom: Theory of mind in old age. Developmental Psychology, 34, 358-362

Harris, P. L. (1991). The work of the imagination. En En A. Whiten (Ed.), Natural theories of mind. Evolution, development and simulation of evereday mindreaning, (pp.283-304). Oxford: Basil Blackwell

Harris, P. L. (1992). Los niños y las emociones. Madrid: Alianza

Harris, P. L., Johnson, C. N., Hutton, D., Andrews, G. Y Cook, T. (1989). Young children's theory of mind and amotion. Cognition and Emotion, 3, 379-400.

Hobson, R. P. (1991). Against the theory of "Theory og Mind. British Journal of Developmental Psychology, 9, 33-51

Hoff.-Ginsberg, E. (1991). Mother-child conversations in different social classes and comunicative settings. Child Development, 62, 782-796

Hughes, C. (1998). Executive function in preschoolers: links whit theory of mind and verbal ability. British Journal of Developmental Psychology, 16, 233-253

Humphrey, N. K. (1983). Counciousness regained. Oxford: Oxford University Press. Trad. Castellana en Fondo de Cultura Económico, México 
Johnson, C. N. (1988). Theory of mind and the structure of concious experience. En J. W. Aistington, P. L. Harris y D. R. Olson (eds.), Developing theories of mind. New York: Cambridge University Press

Leekam, S. (1993). Children's unsdrestanding of intentional falsehood. En A. Whiten (Ed.), Natural theories of mind: evolution, development and simulation of every mindreading, 159-174. Oxford: Basil Blackwell

Leslie, A. M. (1994). ToMM. ToBy, and agency: Core architecture and domain specificity. En L. Hirschfeld y S. Gelman (Eds.), mapping the mind: Domain specificity in cognition and culture. New York: Cambridge University Press.

Lillard, A. S. (1998). Wanting to be it: Children's understanding of intentions underlying pretense. Child Development, 69, 979-991

Mitchell, P. (1997). Introduction to Theory of mind. Children, autism and apes. London: Arnold

Montgomery, D. E. (1997). Wittgenstein's private language argument and children's understanding of mind. Developmental Review, 17, 291-320

Moore,C., Furrow, D., Chiasson, L. y Patriquin, M. (1994). Developmental relationschips between production and comprehension of mental terms. First language, 14, 1-17

Nelson, K. (1981). Social cogntion in a script framework. En J. H. Flavell \& L. Ross (Eds.), Social cognitive development (pp. 97-118). New York: Cambridge University Press

Ninio, A. (1983). Joint book reading as a multiple vocabulary acquisition device. Developmental Psychology, 19, 445-451

Núñez, M. y Rivière, A. (1994). Engaño, intenciones y creencias en el desarrollo y evolución de una psicología natural. Estudios de Psicología, 52, 83-128

Peralta, O. A. y Salsa, A. M. (2001). Interacción materno-infantil con libros con imágenes en dos niveles socioeconómicos. Infancia y Aprendizaje, 24 (3), 325-339

Perner, J. (1991). Understanding the representational mind. Cambridge, MA:MIT Press

Poulin-Dubois, D. (1995). Infants' distinction between animate and inanimate objects: The origins of naïve psychology. EN Ph. Rochat (Ed.), Early social cognition: Under- 
standing others in the first months of life (pp 257-280). Mahwah, NJ, USA: Lawrence Erlbaum Associates, Inc. Publishers

Rabazo, Ma J. y Moreno, J.M. (2005). La composición escrita: aportaciones teóricas y recomendaciones legales para su enseñanza en educación primaria. Revista electrónica de Investigación Psicoeducativa, 6 - 3 (2), 127-157.

Rabazo Méndez, M.J., Moreno Manso, J. M. (2002). El Maestro en Audición y Lenguaje en la Enseñanza Obligatoria. En J. D. Martínez et al. (Eds.), Intervención en Audición y Lenguaje: Casos prácticos, (pp. 49-67). Madrid: EOS.

Riviére, A., Sotillo, M., Sarriá, E. y Núñez, M. (1994). Metarrepresentación, intensionalidad y verbos de referencia mental: un estudio evolutivo. Estudios de Psicología, 51, 21-32

Rogoff, B. (1990). Apprenticeship in thinking: Cognitive development in social contex. New York: Oxford University Press

Russell, J., Jarrold, Ch. y Potel, D. (1994). What makes strategic deception difficult for children -the deception or the strategy? British Journal of Developmental psychology, 9, 331-349

Sigel, I. E. (1997). Modelo de distanciamiento y desarrollo de la competencia representativa. Infancia y Aprendizaje, 78, 13-29

Sorsby, A. J. y Martlew, M. (1991). Representational demands in mother's talk to preschool children in two contexts: picture books reading and modelling task. Journal of Child language, 18, 373-395

Stern, D. N. (1985). The interpersonal world of the infant: A view from psychoanalisis and developmental psychology. New York. Basic Books

Trevarthen, C. (1982). The primary motives for cooperative undersanding. En G. Butterworth y P. Light (Eds.), Social cogniton. Studies of the development of understanding. Brighton: Harvester Press

Villanueva, B., L. (1998): El rechazo entre iguales y la comprensión infantil de los estados mentales. Castellón: Tesis Doctoral

Vygotsky, L. (1978). Mind in society. Cambridge, MA: Harvard Universy Press 
Vygotsky, 1. (1986). Thougth and Language. Cambridge: MIT Press. Edición en castellano, Pensamiento y Lenguaje, Buenos Aires: La Plèyade, 1987

Wellman, H. M. (1990). The child's theory of mind. Cambridge, MIT Press.

Werstch, J. V. (1979). From social interation to higher psychological processes. Human Development, $22,1-22$

Whiten, A. (1991). Natural theories of mind. Evolution, development and simulation of evereday mindreaning. Oxford: Basil Blackwell

Whiten, A. y Byrne, R. W. (1988). Tactical deception in primates. Behavioral and Brain Sciences, 11, 2, 233-273

Wimmer, H. y Perner, J. (1983). Beliefs about beliefs: Representations and constraining function of wrong beliefs in young children's understanding on deception. Cognition, 13, $103-128$

Wood, D., Bruner , J. y Ross, G. (1978). The role of tutoring in problem solving. Journal of Child Psychology and Psychiatry, 17, 89-100

Zaitchik, D. (1991). Is only seeing really believing? Sources of the trae belief in the fasa belief task. Cognitive Development, 6 (1), 91-103

Páginas web

http://herver.musicaviva.com.ar/Hansel.html

http://herver.musicaviva.com.ar/Gatobotas.html

http://herver.musicaviva.com.ar/Caperucita.html

http://herver.musicaviva.com.ar/Blancanieves.html 